\title{
Review
}

\section{Masked Hypertension: A Review}

\author{
Thomas G. PICKERING ${ }^{1)}$, Kazuo EGUCHI ${ }^{1,2)}$, and Kazuomi KARIO ${ }^{2)}$
}

\begin{abstract}
Masked hypertension is defined as a normal blood pressure (BP) in the clinic or office $(<140 / 90 \mathrm{mmHg})$, but an elevated BP out of the clinic (ambulatory daytime BP or home BP $>135 / 85 \mathrm{mmHg}$ ). It may occur in as many as $10 \%$ of the general population, and is important because it is not diagnosed by routine medical examinations, but carries an adverse prognosis, both in terms of increased target organ damage and cardiovascular events. Possible characteristics of individuals with masked hypertension are: relatively young age, male sex, stress or increased physical activity during the daytime, and smoking or drinking habits. Masked hypertension has also been described in treated hypertensive patients (in whom the prognosis is worse than predicted from the clinic pressure) and in children, in whom it may be a precursor of sustained hypertension. It may be suspected in individuals who have a history of occasional high BP readings, but who are apparently normotensive when checked in the office. One practical point is that we should continue to follow such people rather than dismissing them, and encourage out-of-clinic monitoring of BP. This would apply particularly to smokers and those with BP in the prehypertensive range. The potential implications of masked hypertension are huge, but the optimal strategy for detecting the condition in the general population is not yet clear. (Hypertens Res 2007; 30: 479-488)
\end{abstract}

Key Words: ambulatory monitoring, home monitoring, target organ damage, blood pressure measurement

\section{Introduction}

Ever since hypertension was first recognized as a risk factor for cardiovascular disease, it has been identified by measurements made in an office or clinic setting. However, it has been known for more than 50 years that in many individuals these readings tend to be higher than readings taken in other situations. Ayman and Goldshine showed in 1940 (1) that when hypertensive patients had their blood pressure measured at home by a family member, the recorded pressure could be as much as $30 \mathrm{mmHg}$ lower than the readings taken by physicians in their office. At about the same time, Alam and Smirk (2) introduced the concept of casual and basal blood pressure; they showed that when people were allowed to relax for 90 min the blood pressure might be 20 or $30 \mathrm{mmHg}$ lower than when first taken. They defined the casual pressure as the initial pressure taken without any rest, and the basal pressure was the minimum value obtained after a prolonged period of rest. Smirk's idea was that the basal pressure was the better predictor of cardiovascular risk (2). The difference between the casual and basal pressure was termed the supplemental pressure, and was roughly equivalent to what we now refer to as the white coat effect (WCE), as described below. For clinical practice, hypertension has always been defined by an arbitrary threshold level (typically 140/90 $\mathrm{mmHg}$ ) based on casual measurements, above which an individual is identified as being hypertensive, and below which he or she is classified as normotensive. A major implication of this is that anyone who tends to exhibit an exaggerated increase of pressure during a clinic measurement will be labeled as being hypertensive.

For many years, the use of conventional clinic measurements for defining blood pressure status went unchallenged. The first seeds of doubt were sown when Sokolow and his colleagues published the first papers using ambulatory blood pressure monitoring (ABPM) in the 1960s. One of these found that the ambulatory blood pressure was a better predic-

From the ${ }^{1)}$ Center for Behavioral Cardiovascular Health, Division of General Medicine, Columbia University Medical Center, New York, USA; and ${ }^{2}$ Division of Cardiovascular Medicine, Jichi Medical University School of Medicine, Shimotsuke, Japan.

Address for Reprints: Thomas G. Pickering, M.D., D.Phil., Center for Behavioral Cardiovascular Health, Division of General Medicine, Columbia University Medical Center, New York 10032, USA. E-mail: tp2114@columbia.edu

Received January 4, 2007; Accepted in revised form April 20, 2007. 


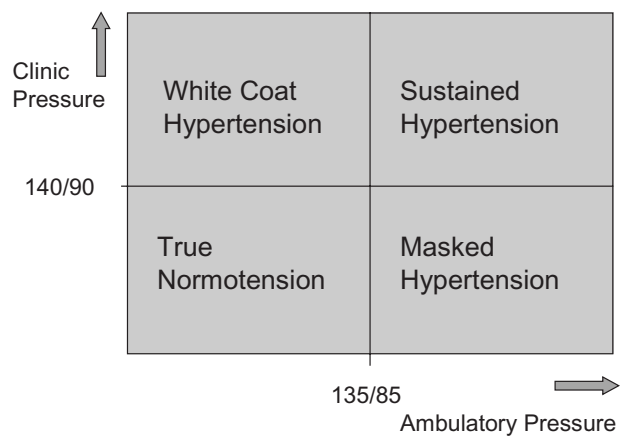

Fig. 1. Classification of blood pressure status according to clinic and ambulatory pressure.

tor of target organ damage than the casual blood pressure (3), and it was subsequently shown that ABPM also was a better predictor of risk of cardiovascular morbidity (4). Since then, the gradual acceptance of ABPM as an improved method for classifying the blood pressure status of patients with suspected hypertension has been driven by the publication of a series of studies that have confirmed that ABPM is a better predictor of target organ damage and risk than conventional clinic measurements.

The use of home- or self-monitoring of blood pressure (SBPM) has been rapidly gaining ground as a more convenient method of out-of-office blood pressure measurement, and while the data are not as comprehensive as with ABPM, there is now good evidence that SBPM also gives better estimates of target organ damage and prognosis than clinic blood pressure (5, 6).

As a result, we now have two independent techniques for measuring blood pressure in clinical practice: conventional clinic or office measurement and out-of-office measurement. For the latter, ABPM is presently the gold standard. This means that we can define hypertension independently by each of the two methods, which together will yield the following four potential groups of patients: 1) those normotensive by both methods (true normotensives); 2) those hypertensive by both methods (true, or sustained hypertensives); 3) those hypertensive by clinic measurement and normotensive by ambulatory measurement (white coat hypertensives); and 4) those normotensive by clinic measurement and hypertensive by ambulatory measurement (see Fig. 1). From a clinical point of view, the first two groups are easy to deal with, since both methods give the same classification. Of more interest are the groups for which there is disagreement. The third group of patients, usually referred to as white coat hypertensives, or less frequently as isolated office hypertensives, have been extensively studied, and are generally accepted as being at relatively low risk of cardiovascular morbidity (7), a view which is consistent with the concept that ambulatory pressure is a better predictor of risk than clinic pressure. However, it must be admitted that not everyone shares the view that white coat hypertension is benign, particularly because there are studies that have shown that it may be associated with some degree of target organ damage.

Up to now, little attention has been given to the fourth group, who were originally given the awkward titles of "reverse white coat hypertensives" or "white coat normotensives" $(8,9)$. If it is true that the ambulatory pressure gives the better classification of risk, this would imply that these people should be regarded as being genuinely hypertensive, as argued below. We have proposed that the phenomenon should be called "masked hypertension," on the grounds that the hypertension is not detected by the routine methods (10).

\section{How Should Masked Hypertension Be Defined?}

The general definition of masked hypertension is a combination of a normal clinic or office blood pressure plus an elevated blood pressure when out of the office. There is little disagreement as to what constitutes a normal clinic pressure, since 140/90 $\mathrm{mmHg}$ is the universally accepted threshold for the majority of hypertensive patients, and it is also accepted that repeated measurements should be used for establishing the definition. What is more problematic is the definition of an elevated out-of-office measurement. Here there are two issues: should the definition be based on ambulatory or selfmonitored readings, and should it be based on measurements made at a particular time of day? The same issues, of course, apply to the definition of white coat hypertension, the mirror image of masked hypertension. Traditionally, white coat hypertension has been defined by the average daytime ambulatory blood pressure, where $135 / 85 \mathrm{mmHg}$ is the most widely accepted threshold (11). This allows for the WCE which is present in the majority of hypertensive patients, and is defined as the difference between the clinic and daytime pressure. It can be positive or negative, and is about $5 \mathrm{mmHg}$ on average in hypertensive patients, such that a clinic pressure of $140 / 90 \mathrm{mmHg}$ is roughly equivalent to a daytime pressure of $135 / 85 \mathrm{mmHg}$.

We have argued previously that white coat hypertension should not be defined solely on the basis of self-measured blood pressure, on the grounds that these readings tend to be taken at times of relative quietude, and may miss a persistently elevated pressure during working hours, for example (12). Nevertheless, as described below, there is increasing evidence that self-measured blood pressure may be a better predictor of risk than clinic pressure (13). The objective for the definition of white coat hypertension is to identify patients who are at low risk of high blood pressure, and thus from whom unnecessary antihypertensive treatment can be withheld.

With masked hypertension, the objective is to identify patients who are either not receiving treatment that would benefit them, or who are being treated inadequately, in both cases because the clinic pressure underestimates the true pres- 
sure. If masked hypertension is regarded as the mirror image of white coat hypertension, the obvious criterion would be a clinic pressure below 140/90 $\mathrm{mmHg}$ plus a daytime pressure above 135/85 mmHg (14). These patients thus have a negative WCE. However, it must be admitted that this definition would not include subjects whose clinic and daytime pressures are normal, but whose nighttime pressures are high. The frequency of this occurrence remains unclear, as does the prognosis. Nevertheless, there is increasing evidence that an elevated nighttime blood pressure may be one of the best predictors of risk (15-19).

\section{Causes of Masked Hypertension}

What factors might lead to masked hypertension? In principle, there are two groups of factors, which are not mutually exclusive. First, the clinic pressure could be relatively low in relation to the ambulatory pressure, or second, there could be factors which selectively raise the ambulatory pressure. A third group includes factors that cannot be specified as exclusively affecting one of the two sets of measurements that are used to define the condition, such as age and gender.

\section{Reduced Clinic Pressure}

Some years ago we observed that in normotensive individuals the clinic pressure was not generally higher than the ambulatory pressure, whereas in hypertensives the clinic pressure was typically higher, even during the hours of work (20). We suggested that the reason for this might be that hypertension is diagnosed on the basis of a high clinic pressure, so any individual who has a tendency for the clinic setting to cause a rise of pressure will be selected out and labeled as being hypertensive.

There has been considerable discussion as to how the WCE should be defined; while there is agreement that the physician-measured pressures are one of the components, there is much less agreement as to what the other should be. For practical purposes, the difference between the clinic and daytime ambulatory pressures has clinical relevance, since both have been related to clinical outcome, and there is a substantial body of literature showing that the WCE when measured in this way does not relate to either clinical outcome or target organ damage (21-24). This measure of the WCE has been criticized on the grounds that the ambulatory blood pressure does not represent a truly "basal" or resting pressure, since it will be affected by factors such as physical and mental activity, and smoking (25). It has therefore been proposed that a better measure would be the blood pressure taken with the patient resting, some time before the physician's reading is taken, and this has been referred to as the "true" WCE. This measure is certainly different from the ambulatory WCE. Although Lantelme et al. (22) found a weak correlation between the two WCE measures $(r=0.31, p=0.004)$, Palatini et al. found none (26). Munakata et al. found that the true
WCE, measured with a Finapres device, correlated with left ventricular mass index in men, but not in women (27), but Lantelme et al. (22) found no relationship in either sex. Palatini et al. (26) found a significant correlation between the true WCE and the blood pressure response to a speech task $(r=0.50, p<0.001)$. Hypertensive patients with a strongly positive WCE (average change of systolic blood pressure $+23.1 \mathrm{mmHg}$ ) did not show higher heart rate changes during a doctor's office visit ( $5.5 \mathrm{vs} .3 .7 / \mathrm{min}$ ) than those with a small WCE $(+3.9 \mathrm{mmHg})$. The average WCE for normotensives was $8.1 \mathrm{mmHg}$ for blood pressure and $0.3 \mathrm{bpm}$ for heart rate. The blood pressure response to a speech task was also greater in hypertensives with a very large WCE $(+60.7 \mathrm{mmHg})$ than in those with a smaller WCE $(+39.7 \mathrm{mmHg})$, but again the heart rate responses were not different. The variability of daytime ambulatory blood pressure was not greater in the patients with the biggest WCE. These studies emphasize several points: first, the WCE is not a manifestation of a generalized increase of blood pressure reactivity or variability, and second, the changes of blood pressure are not closely linked to changes of heart rate, as might be expected if the WCE was simply an exaggerated arousal response.

We have compared the two methods of defining the WCE in hypertensive and normotensive subjects who were evaluated by a series of resting measurements made in the office setting, and by ABPM over a 3-day period. On Day 1, subjects had resting pressures measured in a research laboratory that was not part of the clinic setting, and by a research assistant who was trained to minimize the "medical" aspects of the procedure, and to make no reference to the physician or the clinic. After this the participants were fitted with an ambulatory monitor, which they wore for the next $24 \mathrm{~h}$, at which time they returned to the hospital, but this time the measurements were taken in a medical clinic, and the subjects were informed that readings would be taken by a physician. Readings were taken first in the waiting room, then in the examination room before the physician entered, and then by the physician. The WCE could thus be expressed both as the "ambulatory" WCE and the "true" WCE, the former by taking the daytime average as the resting value, and the latter by taking the resting pressure on Day 1. In normotensive subjects both methods produced a negative WCE. Although there was some increase of blood pressure in the waiting room and the exam room on Day 2 when compared with the Day 1 levels, the physician readings were actually a little lower than the readings taken immediately before, and as we have observed previously, the physician readings were also lower than the daytime average. The latter is not surprising, given that the physician readings were taken with the subject seated at rest, while the ambulatory readings were recorded during normal daily activities. Our study included four groups of subjects: true normotensives, true hypertensives, white coat hypertensives, and masked hypertensives. Two groups showed positive WCE (the true and white coat hypertensives), while two had negative WCE (true normotensives and masked hypertensives). 
Although the absolute values of the ambulatory and true WCE were different, the same groups showed positive and negative WCE by both classifications. Subjects were also asked to self-rate their level of anxiety at the time of each blood pressure measurement. Here again, the same pairing of the four groups was observed. Two scored relatively high on anxiety throughout both days of the study (the true and white coat hypertensives), while two were consistently low (the masked hypertensives and true normotensives). Only the white coat hypertensives showed any marked increase of anxiety at the time of the physician's measurements. Thus subjects who showed a positive WCE tended to score high on anxiety, while those who showed a negative one scored low. We suspect that the explanation for this difference is a labeling phenomenon: subjects who are told that they are hypertensive become more anxious in a clinic setting, and this fosters a positive WCE. This in fact was well described some years ago by Rostrup et al., who studied a group of 32 young Norwegian men who were found to have high blood pressure on screening for military service $(28,29)$. The subjects were divided into two groups, one of which was informed that their pressures were high, while the other was not. Two weeks later they returned for a second series of measurements, which included reactivity testing. The group that had been labeled as being hypertensive showed a persistently higher blood pressure throughout the $45 \mathrm{~min}$ of testing than the uninformed group.

We believe that a positive WCE, such as occurs in white coat hypertension, may in part be a conditioned anxiety response that is relatively specific to the clinic setting. This, of course, does not explain the negative WCE that characterizes masked hypertension. A negative WCE does not necessarily mean that the clinic pressure actually decreases at the time of measurement, although our data are consistent with this, since the resting pressure on Day 1 was actually higher than the physician-measured pressure.

\section{Increased Ambulatory Pressure}

Many factors could selectively elevate the ambulatory pressure. These include lifestyle factors such as smoking, alcohol, physical activity, and mental activity (stress).

\section{Smoking}

We showed many years ago that smokers tend to have high daytime ambulatory pressures (when they are likely to be smoking) in comparison with their clinic pressures (when they are not likely to be smoking) (30). In our study we found a non-significant tendency for masked hypertensives to be smokers (23\% vs. $16 \%)$ or former smokers (43\% vs. $29 \%)$ rather than true normotensives (9). The Second Australian National Blood Pressure Study described above also found that smoking predicted masked hypertension (31). The SHEAF study $(6,32)$, which recruited elderly hypertensive patients on treatment, whose out-of-office pressure was eval- uated by self-monitoring, reported that smoking (classified as active or former) was highest in the masked hypertensive group $(35.0 \%$, as compared to $34.4 \%$ in the uncontrolled hypertensives, $23.6 \%$ in the white coat hypertensives, and $27.6 \%$ in the controlled hypertensives, $p<0.001$ ). The data were also analyzed according to the WCE. In the group with a negative WCE (equivalent to the masked hypertensives), $36.4 \%$ were current or past smokers, while in those with a small WCE the rate was $32.5 \%$, and in those with a positive WCE it was $26.1 \%$. These differences were actually most marked in patients who classified themselves as former smokers $(28.8 \%$ of the negative WCE group, $p<0.001)$, and not significant for current smokers $(7.6 \%)$. This puts into question the explanation offered above, that the reason for the negative WCE in smokers was that they were smoking during the out-of-office blood pressure measurement procedures but not during the clinic measurements, since the differences were most marked in the former smokers. However, it is possible that many of the former smokers were actually still smoking, as the numbers of self-reported current smokers was very low. In a study of 319 clinically normotensive volunteers, Selenta et al. (33) found that $23 \%$ had masked hypertension, and that they tended to be male, past smokers. In our Cornell Worksite study, we found that blood pressure at work tended to be higher in smokers (by $4 / 0.5 \mathrm{mmHg}$ ) and those who drank alcohol (by 3.9/2.9 $\mathrm{mmHg}$ ), although the differences were not quite significant (34). In the Second Australian National Blood Pressure Study (31), which recruited patients over the age of 65 who had a clinic pressure of $160 / 90 \mathrm{mmHg}$ or higher, ABPM was performed in a subset of 713 of them before starting treatment, and in $21 \%$ of these patients there was a reverse WCE (daytime pressure higher than the clinic pressure). Two characteristics of these patients were that they were more likely to be males and smokers.

\section{Alcohol}

The pressor effect of alcohol is another potential contributor to masked hypertension, although the evidence is relatively weak. There was a non-significant trend in the Cornell Worksite study for subjects who drank alcohol to have a high work blood pressure (by $4 / 3 \mathrm{mmHg}$ ), and in a study of Japanese hypertensive patients regular alcohol drinking was associated with masked morning hypertension as detected by self blood pressure monitoring (35).

\section{Physical Activity}

Subjects who are more physically active during the day will tend to have higher daytime pressures (36). An important question arising in this context is whether the nighttime pressure is also raised. In general, hypertensive patients show a sustained elevation of blood pressure over $24 \mathrm{~h}$ relative to normotensive subjects, without much change in the diurnal rhythm of blood pressure. We found that the elevation of blood pressure in masked hypertensives as compared with the blood pressure in true normotensives was $13 / 3 \mathrm{mmHg}$ for the 
Table 1. Prevalence of Masked Hypertension in Various Studies

\begin{tabular}{llrrr}
\hline \multicolumn{1}{c}{ Author } & \multicolumn{1}{c}{ Population } & $N$ & ABP criterion & Prevalence $(\%)$ \\
\hline Imai et al. (47) & Population Ohasama & 969 & $133 / 78$ & 10 \\
Sega et al. (39) & Population PAMELA & 3,200 & $125 / 79$ & 9 \\
Björklund et al. (48) & Population 70-year-old men & 578 & $135 / 85$ & 14 \\
Liu et al. (9) & Healthy volunteers & 234 & $135 / 85$ & 21 \\
Selenta et al. (33) & Healthy volunteers & 319 & $135 / 85$ & 23 \\
\hline
\end{tabular}

ABP, ambulatory blood pressure.

clinic pressure (the average clinic pressure in the masked hypertensives, however, was still well within the normal range of $122 / 80 \mathrm{mmHg}$ ), $17 / 10 \mathrm{mmHg}$ for the work pressure, $18 / 9 \mathrm{mmHg}$ for the home pressure, and $11 / 7 \mathrm{mmHg}$ for the sleep pressure (9). Thus the elevation of blood pressure in masked hypertension appears to be more marked during the day than at night. Experimental evidence that supports the concept that the blood pressure does not have to be raised throughout the day and night to have adverse effects comes from a series of animal experiments performed by Julius et $a l$., who induced a transient neurogenic hypertension in dogs by thigh compression applied for $6 \mathrm{~h}$ a day over a period of 9 weeks (37). This raised the blood pressure for as long as it was applied, but after several months there was no increase in the resting blood pressure. There was, however, an increase of left ventricular mass.

\section{Stress}

A fourth factor may be exposure to stress during the daytime hours. The issue of masked hypertension was discussed in one of our previous papers (38), where we referred to it as occult workplace hypertension. We found that 36 of 467 men in the Cornell Worksite study had masked hypertension, defined as a daytime ambulatory diastolic pressure $>85 \mathrm{mmHg}$, and a clinic pressure $<85 \mathrm{mmHg}$, but the prevalence was no higher in the men exposed to job strain than in those with less stressful jobs. This may be because, as we found in other analyses, job strain has a sustained effect on blood pressure over $24 \mathrm{~h}$, and thus it would be expected to affect the clinic pressure as well as the ambulatory pressure.

\section{Other Factors}

There are several other factors that are associated with masked hypertension but which are not clearly related to either the office or the daytime blood pressure, such as gender and age.

\section{Gender}

A French study called SHEAF (Self measurement of blood pressure at Home in the Elderly, Assessment and Follow-up) (32) recruited 5,211 patients over the age of 60 who were either hypertensive in the clinic or taking antihypertensive medications. About $10 \%$ of these were found to have higher pressures at home than in the clinic, and they appeared to be a relatively high risk group: they tended to be older, and were more likely to be male and to have diabetes or a history of stroke or coronary heart disease than patients whose home and clinic pressures were similar. In previous papers describing both treated and untreated patients with masked hypertension, there is agreement that the percentage of men is higher than women $(33,39-41)$.

\section{Age}

Several population studies have compared clinic and ambulatory blood pressures (42-46). Some have shown daytime pressures to be a little higher than clinic pressures, while others have found the reverse (45). One important finding from an Italian study has been that the ambulatory pressure shows much less increase with age than the clinic pressure (44). And in a Danish study (45) $86 \%$ of men aged 42 years old had daytime pressures higher than the clinic pressure, whereas this was true of only $51 \%$ at the age of 72 years. The WCE is hence more marked in older people, and since masked hypertension is equivalent to a negative WCE, it is reasonable to suppose that masked hypertension would be less prevalent with increasing age.

\section{Prevalence of Masked Hypertension}

Another important issue is the prevalence of masked hypertension. While there are no definitive data, the available information in three population-based studies is disturbing (see Table 1).

The first was the Ohasama study (47), conducted in a small Japanese town, which reported that $10.4 \%$ of subjects with normal screening blood pressures had ambulatory pressures that were in the "borderline hypertensive" range (ambulatory systolic blood pressure 133-144 $\mathrm{mmHg}$, and diastolic blood pressure $78-85 \mathrm{mmHg}$ ), and another $3.2 \%$ in the definitely hypertensive range. The second was the PAMELA study quoted above, which found masked hypertension in $9 \%$ of subjects (39); similarly, Selenta et al. (33) found it in 23\% of their "normal" volunteers. However, the PAMELA study used a lower upper limit of normal for the 24-h pressure (125/ $79 \mathrm{mmHg}$ ) compared to most other studies, and it could be argued that if a higher level had been used, the number of masked hypertensives would be smaller. But even if the prev- 
alence was only $5 \%$, this number applies to the whole population, not just the population of hypertensives, so in the case of the United States this might amount to 15 million people. The third was the study of Björklund et al. of a cohort of 70-yearold Swedish men who were all born in the same year. Out of a total of 578 men, $33 \%$ were normotensive by clinic measurements, and of these, $14 \%$ met the criteria of masked hypertension (48).

Two other studies that were not based on population samples reported a higher prevalence of masked hypertension. The first was our own study (9), where we found a prevalence of $21 \%$, and the second was a study of 319 clinically normotensive volunteers reported by Selenta et al. (33). The subjects were evaluated with 5 clinic measurements and 12-h daytime ABPM, and 23\% had masked hypertension, defined as a daytime blood pressure above $135 / 85 \mathrm{mmHg}$. Subjects with masked hypertension tended to be male, past smokers, and older, and had consumed more alcohol. In 558 clinically normotensive healthy workers in Japan, the prevalence of masked hypertension as evaluated by home blood pressure monitoring was $7.2 \%$ (systolic) and 8.7\% (diastolic) (49).

Masked hypertension has also been described in children. In one study of 592 children (mean age 10.2 years) reported by Lurbe et al. (50), 45 (7.6\%) children had masked hypertension. When compared with normotensives, the children with masked hypertension had higher ambulatory pulse rates, were more obese, and were more likely to have a parental history of hypertension at baseline. During a median follow-up period of 37 months, among 34 subjects with masked hypertension, 18 became normotensive, 13 had persistent masked hypertension, and 3 developed sustained hypertension. Patients with persistent masked hypertension $(n=17)$ or those who progressed from masked to sustained hypertension $(n=3)$ had a higher left ventricular mass index (34.9 vs. $29.6 \mathrm{~g} / \mathrm{m}^{2.7}$; $p=0.023$ ). In another study, masked hypertension was found in $9.4 \%$ of 85 children and was only present in children who were not obese. It was associated with a significantly higher left ventricular mass index than in normotensives (51). Thus masked hypertension appears to be a phenomenon that should be taken seriously in children as well as adults.

\section{Diagnosis and Reproducibility of Masked Hypertension}

There is also support for the concept of an adverse prognosis in patients with masked hypertension from home blood pressure data. One of the first publications on this subject was performed in 1993 by Shahab et al., who measured home and clinic blood pressure in a group of healthy young subjects (52). About $10 \%$ of these were classified as "hypertensive at home," with normal clinic pressures and raised home pressures. This group was overweight and had high insulin and low high-density lipoprotein (HDL) cholesterol levels, suggesting that they might be at high risk.

One of the problems when masked hypertension is diag- nosed by home monitoring is that blood pressure tends to be higher in the morning than in the evening, and there is no consensus about which time of day the measurement should be performed. Stergiou et al. (53) used the average duplicate data of morning ( 7 to 10 AM) and evening (6 to 9 PM) measurements, and concluded that home blood pressure can be used as a substitute for ABPM to diagnose masked hypertension. In the PAMELA study (39), single readings taken at 7 $\mathrm{AM}$ and $7 \mathrm{PM}$ were averaged. In the J-HOME study, blood pressure was measured once each morning within $1 \mathrm{~h}$ of waking, before taking antihypertensive drugs, and once every evening just before bedtime (54). However, Mallion et al. reported that three measurements at each of two visits are necessary to diagnose masked hypertension properly using office blood pressure, and three measurements in the morning and again in the evening over 2 days are needed for accurate diagnosis by home monitoring (55).

One of the most important issues in masked hypertension is the reproducibility of measurement. At the present time, there are very few data that have systematically investigated this matter. One exception is the study of Lurbe et al., who reported that the blood pressure pattern of masked hypertension persisted in $40 \%$ of children over a 3 -year period (50).

There is a suggestion that masked hypertension may be a precursor of true hypertension. In the ongoing HARVEST study (56), Palatini et al. (26) have been following a group of young subjects who were initially identified as being hypertensive by clinic criteria, with both clinic and ambulatory blood pressure measurements. When these measurements were repeated 3 months later, $28 \%$ of the originally hypertensive group had normal clinic pressures. Of these, half had normal ambulatory pressures as well, while half (the masked hypertensives) had elevated ambulatory pressures. Over a 6year follow-up, the masked hypertensives were twice as likely to develop sustained hypertension as the true normotensives. Thus more data are needed to establish the short-term reproducibility of masked hypertension, but there is some evidence that when measurements are made over longer periods of time, masked hypertension may in some cases be a precursor of sustained hypertension.

\section{Target Organ Damage and Prognosis}

The first study to look at the issue of target organ damage was our publication of 1999 (9), in which we showed that a group of patients with masked hypertension had a higher left ventricular mass and more carotid atherosclerosis than true normotensives, and thus were similar to true hypertensives. The LVMI was $73 \mathrm{~g} / \mathrm{m}^{2}$ in the true normotensives, $86 \mathrm{~g} / \mathrm{m}^{2}$ in the masked hypertensives, and $90 \mathrm{~g} / \mathrm{m}^{2}$ in the true hypertensives. Carotid plaque was present in $15 \%$ of true normotensives, and in $28 \%$ of both the masked and true hypertensives. This was the first finding to suggest that masked hypertensives may be at increased risk of cardiovascular morbidity. More recently, an analysis of the PAMELA data (39), a population study of 
3,200 Italians, classified the subjects in the four groups that we have described above. Individuals with treated hypertension were excluded from this analysis; $67 \%$ were true normotensives, $12 \%$ true hypertensives, $12 \%$ white coat hypertensives, and 9\% masked hypertensives. The average clinic pressure in the masked hypertensives was 129/84 $\mathrm{mmHg}$, which while still within the normal range, was higher than that of the true normotensives $(112 / 77 \mathrm{mmHg})$. The clinic pressure of the true hypertensives was $159 / 98 \mathrm{mmHg}$. The left ventricular mass index in the masked hypertensives $\left(91 \mathrm{~g} / \mathrm{m}^{2}\right)$ was higher than that in the true normotensives (79 $\left.\mathrm{g} / \mathrm{m}^{2}\right)$, and similar to that in the true hypertensives $\left(94 \mathrm{~g} / \mathrm{m}^{2}\right)$. In a recent Japanese study, carotid intima-media thickness (IMT) and pulse wave velocity in patients with untreated masked hypertension (defined by home blood pressure) were higher than in normotensive subjects (41).

There are three papers which have described the cardiovascular prognosis of subjects with untreated masked hypertension. Ohkubo et al. reported that in the Ohasama study cardiovascular mortality and stroke morbidity were increased to the same degree in both masked and sustained hypertension in comparison with normotensive subjects (40).

Björklund et al. reported that isolated ambulatory (masked) hypertension was a predictor of cardiovascular morbidity in 578 untreated 70-year-old men independent of established risk factors (48). In the PAMELA study, a population-based study in which only about $10 \%$ of the subjects were treated hypertensives, Mancia et al. reported that subjects with masked hypertension had a higher prevalence of cardiovascular and all-cause mortality (57) compared with the subjects with normal office and 24-h blood pressure.

\section{Masked Hypertension in Treated Patients}

The issue with treated patients is somewhat different. By definition, these are people in whom a diagnosis of hypertension has already been made, so screening is not an issue.

However, masked hypertension is of potential importance in these patients, because the clinic blood pressure may give a false impression that the blood pressure is adequately controlled. Two important issues here are the prevalence in the population of hypertensive patients who are on treatment, and the impact on prognosis.

\section{Prevalence}

The prevalence of masked hypertension in treated hypertensive subjects was $9.4 \%$ when assessed with home blood pressure monitoring in the SHEAF study (6), and $19 \%$ in the JHOME study (54). The number of home measurements was smaller in the J-HOME study, which could be one reason for the difference. In a survey of patients attending a hypertension clinic, all of whom underwent ambulatory monitoring, Pierdomenico et al. reported that one-third of patients whose blood pressure was controlled by clinic criteria (clinic pres- sure $<140 / 90 \mathrm{mmHg}$ ) had masked hypertension (daytime pressure $<135 / 85 \mathrm{mmHg}$ ) (58).

\section{Prognosis}

In treated hypertensives it has also been observed that patients whose ambulatory pressure is high in relationship to their clinic pressure are more likely to have increased left ventricular mass than those whose ambulatory pressure is the same or lower than the clinic pressure $(59,60)$. These findings are consistent with the view that when there is a discrepancy between clinic and ambulatory pressure, it is the latter that is most closely related to target organ damage. A study by Schillaci et al. (59) included 395 treated hypertensives who were evaluated with clinic and ambulatory monitoring. There was one group that had high ABPM in reference to the clinic pressure, and these patients had greater left ventricular mass and a more concentric pattern of left ventricular hypertrophy than the patients whose ambulatory blood pressure was closer to the clinic pressure. However, they would not strictly be classified as having masked hypertension since the clinic pressures were also elevated (144/89 $\mathrm{mmHg})$.

In the SHEAF study described above (6), which recruited elderly hypertensives on treatment, the hazard ratio for cardiovascular events was 2.06 (95\% confidence interval [CI], $1.22-3.47)$ in patients with masked hypertension, with the patients whose home and clinic blood pressure were both normal as the referent. Pierdomenico et al. (58) reported the occurrence of fatal and nonfatal cardiovascular events in treated hypertensive subjects classified by the cut off values of 140/90 $\mathrm{mmHg}$ for clinic blood pressure and 135/85 $\mathrm{mmHg}$ for daytime blood pressure with ABPM. The event-rate per 100 patient-years was 2.42 in the patients with masked hypertension, and 0.87 in those with controlled hypertension. Thus all three studies suggest that masked hypertension in treated patients carries a poor prognosis.

In the Ohasama study, treated masked hypertensive subjects $(n=74)$ were separately analyzed and an increased hazard ratio for cardiovascular disease mortality/stroke morbidity (heart rate $=1.62 \mathrm{bpm} ; 95 \%$ CI, 0.86-3.07) was observed compared with sustained normotensive subjects (40).

\section{Implications of Masked Hypertension}

It seems clear that masked hypertension should be taken seriously, and is a phenomenon worthy of further investigation. If it is accepted that ambulatory blood pressure gives a better prognosis than clinic blood pressure, and that the correlation between the two is only moderate, it is logical to propose that there will be a significant number of people who are truly hypertensive, but in whom the diagnosis is missed by clinic measurement. But how frequently this phenomenon occurs, and how such individuals should be identified, remains a mystery. Clearly, we cannot argue for screening of the gen- 
eral population, but there are many patients who are referred for suspected hypertension who turn out to have normal clinic pressures on repeat testing. Perhaps some of them would benefit from ambulatory monitoring to rule out masked hypertension.

The implications of the concept that there are a substantial number of people in the general population who have undiagnosed and untreated hypertension which puts them at increased risk of cardiovascular disease require serious consideration. Thus, it would significantly change the number of people in the population who have "hypertension" that requires treatment. On the basis of clinic pressures the official estimate from surveys performed in the United States, such as NHANES, is that there are about 65 million hypertensive people (61). If we accept that white coat hypertension is present in $15 \%$ of these, we would reduce the number at risk by nearly 10 million, leaving 55 million who are hypertensive by both clinic and ambulatory criteria. But if we also include the masked hypertensives, who as we saw above may comprise as many as $10 \%$ of the total population, we would need to add $10 \%$ of 300 million, which is another 30 million people. Thus the total population with ambulatory hypertension would increase to 85 million. Looked at another way, the current clinic-based definition of hypertension correctly identifies 55 million people who are truly hypertensive, but incorrectly identifies or fails to identify another 40 million, either because of false positives (10 million white coat hypertensives) or false negatives (30 million masked hypertensives). Thus for every two people that are classified properly, there is another one person who it misclassified - not a very good record for a diagnostic test.

What are the practical implications of masked hypertension? It would clearly be inappropriate to say that everyone should be screened with ambulatory blood pressure monitoring. Before we translate these considerations into clinical practice we need more prospective data to show that masked hypertension does indeed increase cardiovascular risk. Ideally, we should also like to know if treating such patients lowers their risk. We also need better means of identifying such individuals, such as the role of home monitoring. It may be that masked hypertension is a precursor of sustained hypertension. We see many patients who have a history of occasional high blood pressure readings, who are normotensive when we check them in our office. One practical point is that we should continue to follow such people rather than dismissing them, and encourage out-of-office monitoring of blood pressure. This would apply particularly to smokers.

There are also important implications for treated patients. Whether or not patients with controlled clinic blood pressure but uncontrolled out-of-office pressures should be labeled as having masked hypertension is debatable, since the diagnosis of hypertension has already been made, but the implications are the same, namely that in these patients the conventionally recorded clinic pressure underestimates their risk. Their existence strengthens the case for recommending home blood pressure monitoring as part of the routine care of hypertensive patients.

\section{References}

1. Ayman P, Goldshine AD: Blood pressure determinations by patients with essential hypertension I. The difference between clinic and home readings before treatment. Am J Med Sci 1940; 200: 465-474.

2. Alam M, Smirk FM: Casual and basal blood pressures. I. In British and Egyptian men. Brit Heart J 1943; 5: 152-160.

3. Sokolow M, Werdegar D, Kain HK, Hinman AT: Relationship between level of blood pressure measured casually and by portable recorders and severity of complications in essential hypertension. Circulation 1966; 34: 279-298.

4. Perloff D, Sokolow M, Cowan R: The prognostic value of ambulatory blood pressures. JAMA 1983; 249: 2792-2798.

5. Imai Y, Ohkubo T, Tsuji I, et al: Prognostic value of ambulatory and home blood pressure measurements in comparison to screening blood pressure measurements: a pilot study in Ohasama. Blood Press Monit 1996; 1 (Suppl 2): S51S58.

6. Bobrie G, Chatellier G, Genes N, et al: Cardiovascular prognosis of "masked hypertension" detected by blood pressure self-measurement in elderly treated hypertensive patients. JAMA 2004; 291: 1342-1349.

7. Verdecchia P, Schillaci G, Borgioni C, Ciucci A, Porcellati C: White-coat hypertension: not guilty when correctly defined. Blood Press Monit 1998; 3: 147-152.

8. Mancia G: Reversed white-coat hypertension: definition, mechanisms and prognostic implications. $J$ Hypertens 2002; 20: 579-581.

9. Liu JE, Roman MJ, Pini R, Schwartz JE, Pickering TG, Devereux RB: Cardiac and arterial target organ damage in adults with elevated ambulatory and normal office blood pressure. Ann Intern Med 1999; 131: 564-572.

10. Pickering TG, Davidson K, Gerin W, Schwartz JE: Masked hypertension. Hypertension 2002; 40: 795-796.

11. Verdecchia P, Schillaci G, Boldrini F, Zampi I, Porcellati C: Variability between current definitions of 'normal' ambulatory blood pressure. Implications in the assessment of white coat hypertension. Hypertension 1992; 20: 555-562.

12. Pickering TG, Coats A, Mallion JM, Mancia G, Verdecchia P: Blood Pressure Monitoring. Task force V: white-coat hypertension. Blood Press Monit 1999; 4: 333-341.

13. Tachibana R, Tabara Y, Kondo I, Miki T, Kohara K: Home blood pressure is a better predictor of carotid atherosclerosis than office blood pressure in community-dwelling subjects. Hypertens Res 2004; 27: 633-639.

14. Japanese Society of Hypertension: Japanese Society of Hypertension Guidelines for the Management of Hypertension (JSH 2004). Hypertens Res 2006; 29 (Suppl): S1S105.

15. Kario K, Pickering TG, Matsuo T, Hoshide S, Schwartz JE, Shimada K: Stroke prognosis and abnormal nocturnal blood pressure falls in older hypertensives. Hypertension 2001; 38: $852-857$.

16. Sega R, Facchetti R, Bombelli M, et al: Prognostic value of ambulatory and home blood pressures compared with office 
blood pressure in the general population: follow-up results from the Pressioni Arteriose Monitorate e Loro Associazioni (PAMELA) study. Circulation 2005; 111: 1777-1783.

17. Kikuya M, Ohkubo T, Asayama K, et al: Ambulatory blood pressure and 10-year risk of cardiovascular and noncardiovascular mortality: the Ohasama study. Hypertension 2005; 45: 240-245.

18. Davidson MB, Hix JK, Vidt DG, Brotman DJ: Association of impaired diurnal blood pressure variation with a subsequent decline in glomerular filtration rate. Arch Intern Med 2006; 166: 846-852.

19. Hoshide S, Ishikawa J, Eguchi K, Ojima T, Shimada K, Kario K: Masked nocturnal hypertension and target organ damage in hypertensives with well-controlled self-measured home blood pressure. Hypertens Res 2007; 30: 143 149.

20. James GD, Pickering TG, Yee LS, Harshfield GA, Riva S, Laragh JH: The reproducibility of average ambulatory, home, and clinic pressures. Hypertension 1988; 11 (6 Pt 1): 545-549.

21. Guida L, Iannuzzi R, Crivaro M, et al: Clinic-daytime blood pressure difference and cardiovascular damage. J Hypertens 1999; 17: 331-337.

22. Lantelme P, Milon H, Vernet M, Gayet C: Difference between office and ambulatory blood pressure or real white coat effect: does it matter in terms of prognosis? J Hypertens 2000; 18: 383-389.

23. Kristensen KS, Hoegholm A, Bang LE, Gustavsen PH, Poulsen CB: No impact of blood pressure variability on microalbuminuria and left ventricular geometry: analysis of daytime variation, diurnal variation and 'white coat' effect. Blood Press Monit 2001; 6: 125-131.

24. Verdecchia P, Schillaci G, Borgioni C, Ciucci A, Porcellati C: Prognostic significance of the white coat effect. Hypertension 1997; 29: 1218-1224.

25. Parati G, Ulian L, Santucciu C, Omboni S, Mancia G: Difference between clinic and daytime blood pressure is not a measure of the white coat effect. Hypertension 1998; 31: 1185-1189.

26. Palatini $\mathrm{P}$, Palomba D, Bertolo $\mathrm{O}$, et al: The white-coat effect is unrelated to the difference between clinic and daytime blood pressure and is associated with greater reactivity to public speaking. J Hypertens 2003; 21: 545-553.

27. Munakata M, Saito $Y$, Nunokawa T, Ito N, Fukudo S, Yoshinaga K: Clinical significance of blood pressure response triggered by a doctor's visit in patients with essential hypertension. Hypertens Res 2002; 25: 343-349.

28. Rostrup M, Kjeldsen SE, Eide IK: Awareness of hypertension increases blood pressure and sympathetic responses to cold pressor test. Am J Hypertens 1990; 3 (12 Pt 1): 912917.

29. Rostrup M, Mundal HH, Westheim A, Eide I: Awareness of high blood pressure increases arterial plasma catecholamines, platelet noradrenaline and adrenergic responses to mental stress. J Hypertens 1991; 9: 159-166.

30. Mann SJ, James GD, Wang RS, Pickering TG: Elevation of ambulatory systolic blood pressure in hypertensive smokers. A case-control study. JAMA 1991; 265: 2226-2228.

31. Wing LM, Brown MA, Beilin LJ, Ryan P, Reid CM: 'Reverse white-coat hypertension' in older hypertensives. $J$
Hypertens 2002; 20: 639-644.

32. Bobrie G, Genes N, Vaur L, et al: Is "isolated home" hypertension as opposed to "isolated office" hypertension a sign of greater cardiovascular risk? Arch Intern Med 2001; 161: 2205-2211.

33. Selenta C, Hogan BE, Linden W: How often do office blood pressure measurements fail to identify true hypertension? An exploration of white-coat normotension. Arch Fam Med 2000; 9: 533-540.

34. Schnall PL, Schwartz JE, Landsbergis PA, Warren K, Pickering TG: Relation between job strain, alcohol, and ambulatory blood pressure. Hypertension 1992; 19: 488-494.

35. Ishikawa J, Kario K, Eguchi K, et al, J-MORE group: Regular alcohol drinking is a determinant of masked morning hypertension detected by home blood pressure monitoring in medicated hypertensive patients with well-controlled clinic blood pressure: the Jichi Morning Hypertension Research (J-MORE) study. Hypertens Res 2006; 29: 679686.

36. Leary AC, Donnan PT, MacDonald TM, Murphy MB: The influence of physical activity on the variability of ambulatory blood pressure. Am J Hypertens 2000; 13: 1067-1073.

37. Julius S, Li Y, Brant D, Krause L, Buda AJ: Neurogenic pressor episodes fail to cause hypertension, but do induce cardiac hypertrophy. Hypertension 1989; 13: 422-429.

38. Belkic KL, Schnall PL, Landsbergis PA, et al: Hypertension at the workplace - an occult disease? The need for work site surveillance. Adv Psychosom Med 2001; 22: 116138.

39. Sega R, Trocino G, Lanzarotti A, et al: Alterations of cardiac structure in patients with isolated office, ambulatory, or home hypertension: data from the general population (Pressione Arteriose Monitorate e Loro Associazioni [PAMELA] Study). Circulation 2001; 104: 1385-1392.

40. Ohkubo T, Kikuya M, Metoki $\mathrm{H}$, et al: Prognosis of "masked" hypertension and "white-coat" hypertension detected by 24-h ambulatory blood pressure monitoring 10year follow-up from the Ohasama study. $\mathrm{J} \mathrm{Am} \mathrm{Coll} \mathrm{Cardiol}$ 2005; 46: 508-515.

41. Matsui Y, Eguchi K, Ishikawa J, Hoshide S, Shimada K, Kario K: Subclinical arterial damage in untreated masked hypertensive subjects detected by home blood pressure measurement. Am J Hypertens 2007; 20: 385-391.

42. Imai Y, Nagai K, Sakuma M, et al: Ambulatory blood pressure of adults in Ohasama, Japan. Hypertension 1993; 22: 900-912.

43. O'Brien E, Murphy J, Tyndall A, et al: Twenty-four-hour ambulatory blood pressure in men and women aged 17 to 80 years: the Allied Irish Bank Study. J Hypertens 1991; 9: 355-360.

44. Mancia G, Sega R, Bravi C, et al: Ambulatory blood pressure normality: results from the PAMELA study. J Hypertens 1995; 13 (12 Pt 1): 1377-1390.

45. Rasmussen SL, Torp-Pedersen C, Borch-Johnsen K, Ibsen $\mathrm{H}$ : Normal values for ambulatory blood pressure and differences between casual blood pressure and ambulatory blood pressure: results from a Danish population survey. J Hypertens 1998; 16: 1415-1424.

46. Manning G, Rushton L, Millar-Craig MW: Twenty-four hour ambulatory blood pressure: a sample from a normal 
British population. J Hum Hypertens 1998; 12: 123-127.

47. Imai Y, Tsuji I, Nagai K, et al: Ambulatory blood pressure monitoring in evaluating the prevalence of hypertension in adults in Ohasama, a rural Japanese community. Hypertens Res 1996; 19: 207-212.

48. Björklund K, Lind L, Zethelius B, Andren B, Lithell H: Isolated ambulatory hypertension predicts cardiovascular morbidity in elderly men. Circulation 2003; 107: 1297-1302.

49. Kawabe H, Saito I, Saruta T: Status of home blood pressure measured in morning and evening: evaluation in normotensives and hypertensives in Japanese urban population. Hypertens Res 2005; 28: 491-498.

50. Lurbe E, Torro I, Alvarez V, et al: Prevalence, persistence, and clinical significance of masked hypertension in youth. Hypertension 2005; 45: 493-498.

51. Stabouli S, Kotsis V, Toumanidis S, Papamichael C, Constantopoulos A, Zakopoulos N: White-coat and masked hypertension in children: association with target-organ damage. Pediatr Nephrol 2005; 20: 1151-1155.

52. Shahab ST, Gudbrandsson T, Jamerson K, Julius S: Isolated "home hypertension" in Tecumseh, Michigan. Croat Med J 1993; 34: 325-331.

53. Stergiou GS, Salgami EV, Tzamouranis DG, Roussias LG: Masked hypertension assessed by ambulatory blood pressure versus home blood pressure monitoring: is it the same phenomenon? Am J Hypertens 2005; 18: 772-778.

54. Obara T, Ohkubo T, Kikuya M, et al: Prevalence of masked uncontrolled and treated white-coat hypertension defined according to the average of morning and evening home blood pressure value: from the Japan Home versus Office
Measurement Evaluation Study. Blood Press Monit 2005; 10: 311-316.

55. Mallion JM, Genes N, Vaur L, et al: Detection of masked hypertension by home blood pressure measurement: is the number of measurements an important issue? Blood Press Monit 2004; 9: 301-305.

56. Palatini P, Winnicki M, Santonastaso M, et al: Prevalence and clinical significance of isolated ambulatory hypertension in young subjects screened for stage 1 hypertension. Hypertension 2004; 44: 170-174.

57. Mancia G, Facchetti R, Bombelli M, Grassi G, Sega R: Long-term risk of mortality associated with selective and combined elevation in office, home, and ambulatory blood pressure. Hypertension 2006; 47: 846-853.

58. Pierdomenico SD, Lapenna D, Bucci A, et al: Cardiovascular outcome in treated hypertensive patients with responder, masked, false resistant, and true resistant hypertension. $\mathrm{Am}$ J Hypertens 2005; 18: 1422-1428.

59. Schillaci G, Verdecchia P, Sacchi N, et al: Clinical relevance of office underestimation of usual blood pressure in treated hypertension. Am J Hypertens 2000; 13 (5 Pt 1): 523-528.

60. Cuspidi C, Lonati L, Sampieri L, et al: Prevalence of target organ damage in treated hypertensive patients: different impact of clinic and ambulatory blood pressure control. $J$ Hypertens 2000; 18: 803-809.

61. Burt VL, Whelton P, Roccella EJ, et al: Prevalence of hypertension in the US adult population. Results from the Third National Health and Nutrition Examination Survey, 1988-1991. Hypertension 1995; 25: 305-313. 